\title{
PALYNOLOGICAL ANALYSIS OF 228 OUTCROP SAMPLES FROM THE KENAI, SELDOVIA, AND TYONEK QUADRANGLES, COOK INLET REGION, ALASKA
}

by

Pierre Zippi and Andrea M. Loveland

\author{
Released by
}

STATE OF ALASKA

DEPARTMENT OF NATURAL RESOURCES

Division of Geological \& Geophysical Surveys

3354 College Road

Fairbanks, Alaska 99709-3707 



\title{
Palynological Analysis of $\mathbf{2 2 8}$ Outcrop Samples from the Kenai, Seldovia, and Tyonek Quadrangles, Cook Inlet Region, Alaska
}

\author{
by \\ Pierre Zippi \\ Biostratigraphy.com, LLC
}

and

Andrea M. Loveland

Alaska Divison of Geological \& Geophysical Surveys, 3354 College Rd., Fairbanks, AK 99709-3707

\begin{abstract}
INTRODUCTION
- Paul Decker - PLD

- Bob Gillis - BG

- Trystan Herriott - TMH

- Dave LePain - DL

- Dave Mauel - DJM

- Jacob Mongrain - JRM

- Marwan Wartes - MAW
\end{abstract}

The palynological samples included in this Raw Data File were collected as part of the Alaska Department of Natural Resources' (Alaska Division of Geological \& Geophysical Surveys [DGGS] and Alaska Division of Oil \& Gas [DOG]) Cook Inlet Basin Analysis program. The focus of this program is to evaluate the stratigraphic trap potential and reservoir quality of coal-bearing Cenozoic strata in upper Cook Inlet basin and the reservoir quality of Mesozoic strata throughout the basin. The samples included in this report were collected in the Tyonek, Kenai, and Seldovia quadrangles from September 2006 through July 2010. All samples in this report were processed by Russ Harms of Global Geolab Limited in Medicine Hat, Alberta, Canada. Prepared slides of recovered palynomorphs were analyzed by Dr. Pierre Zippi (Biostratigraphy.com, LLC). Sample collectors include Paul Decker (DOG), Emily Finzel (Purdue University), Bob Gillis (DGGS), Trystan Herriott (DGGS), Dave LePain (DGGS), Dave Mauel (DGGS), Jacob Mongrain (UAF and DGGS), and Marwan Wartes (DGGS). Sample numbers include year collected, collector initials, field location number, and sample location in measured stratigraphic section, if tied to a measured section. Collector initials include:

An example of the sample numbering scheme follows:

- $\quad$ 07DL085-35 Indicates the sample was collected in 2007 by Dave LePain at his field location 85. In this case the sample is tied to a measured stratigraphic section and the last number shows its position in meters above the base of the section.

Another example includes:

- $\quad$ 08BG184b Indicates the sample was collected by Bob Gillis in 2008 at his field location 184. The letter " $b$ " indicates that other samples (not for palynological analysis) were collected at that location.

The following sample numbers deviate from the numbering convention outlined above:

- $\mathrm{ST} 1$ -

- $\mathrm{TY}-$

- $\mathrm{BG1}-$

These samples were collected by Emily Finzel (Purdue University). ST1=Sterling Formation; BG1 = Beluga Formation; TY=Tyonek Formation

Figure 1 is an overview map showing the distribution of samples analyzed. Figures $2-5$ offer a more detailed look at sample locations. Table 1 summarizes the palynological results for each sample. 


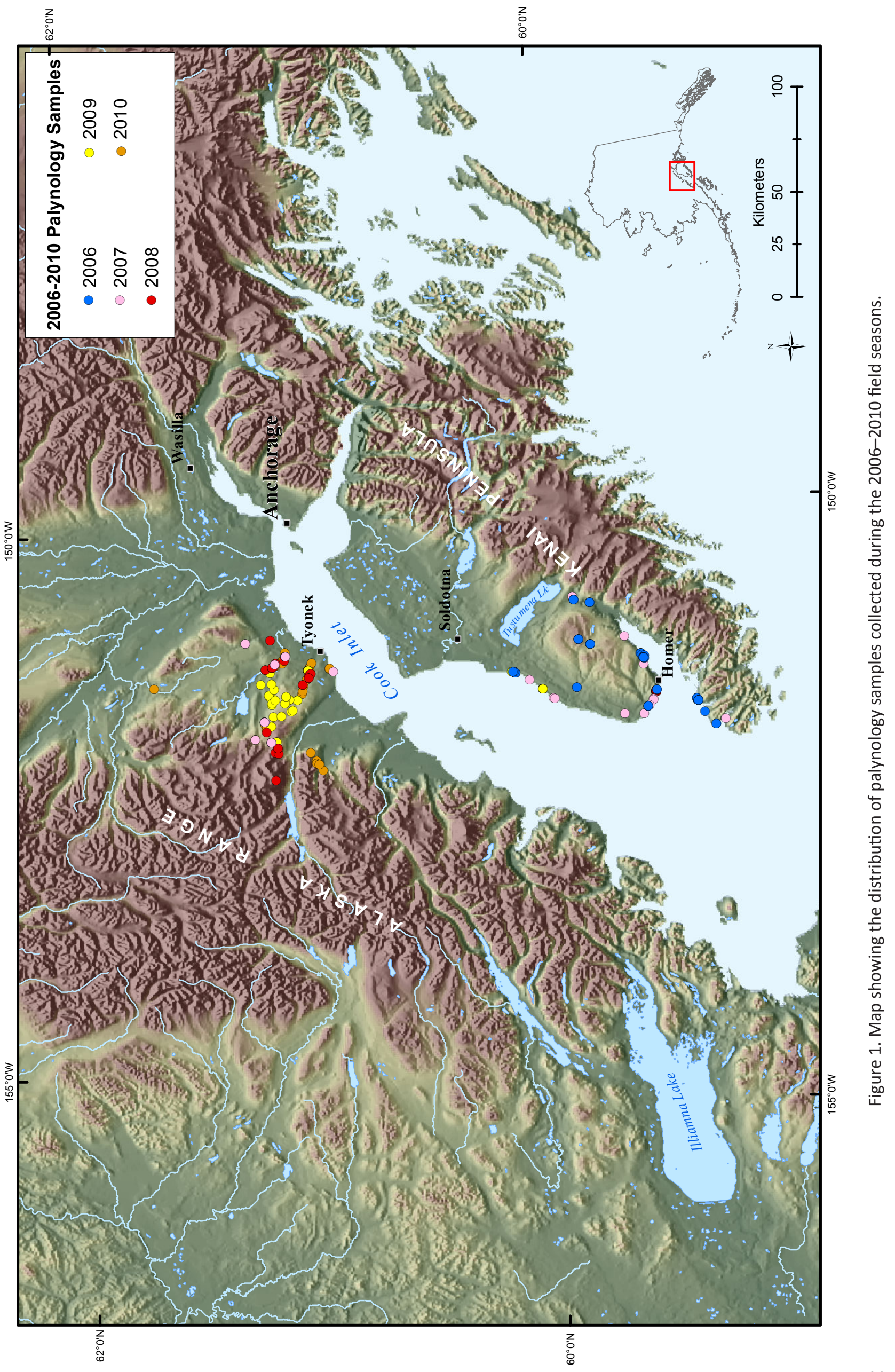

RDF 2012-1 


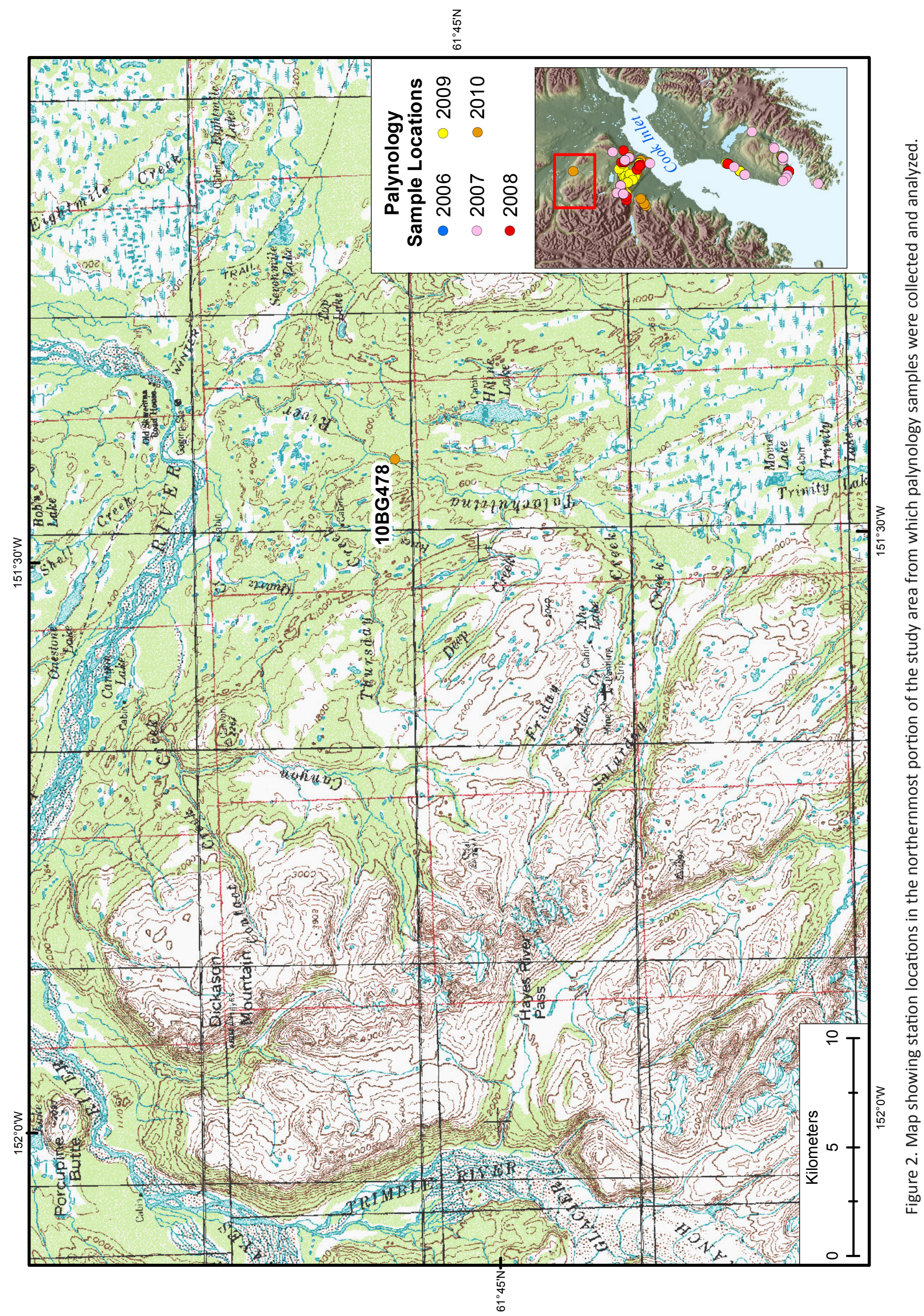




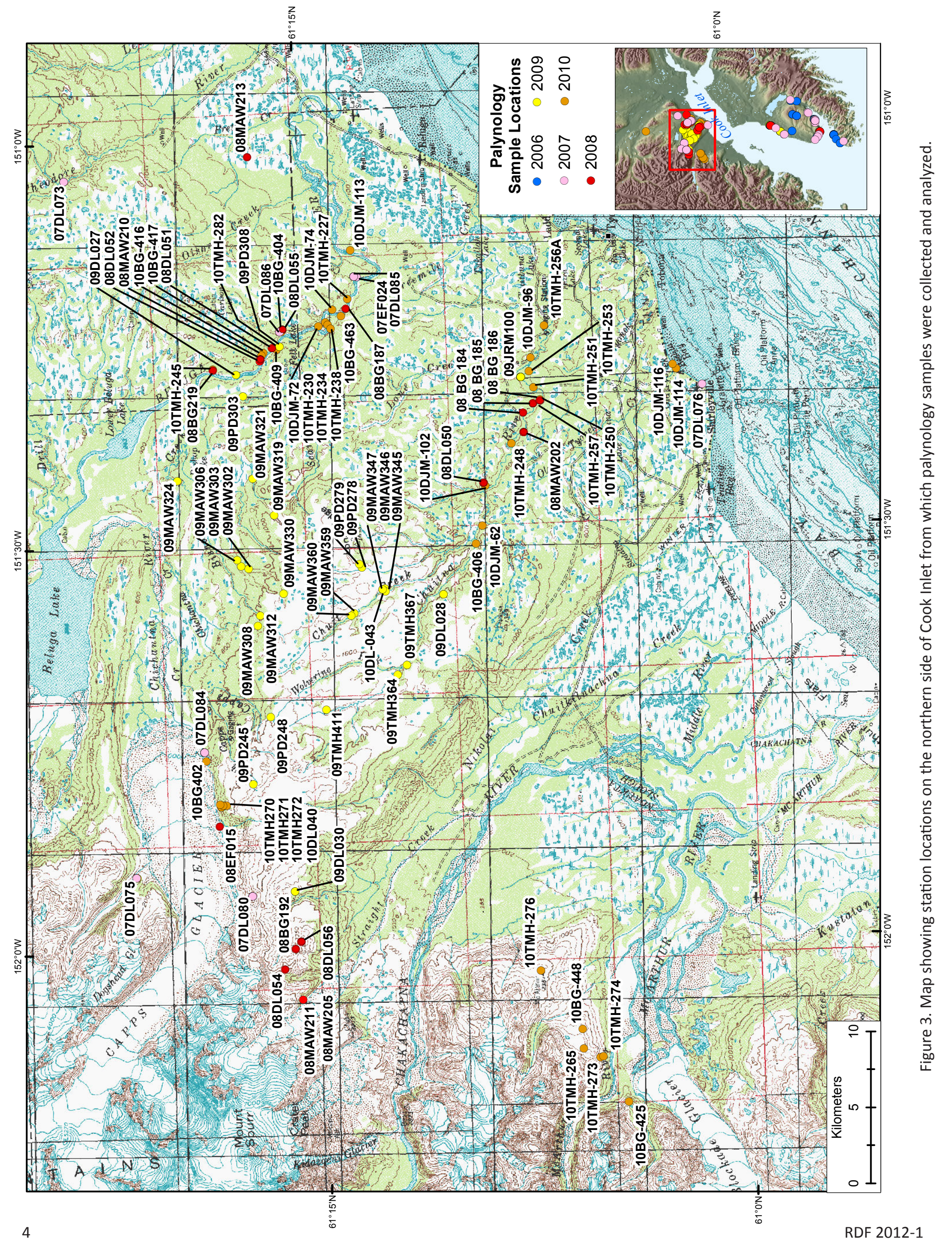




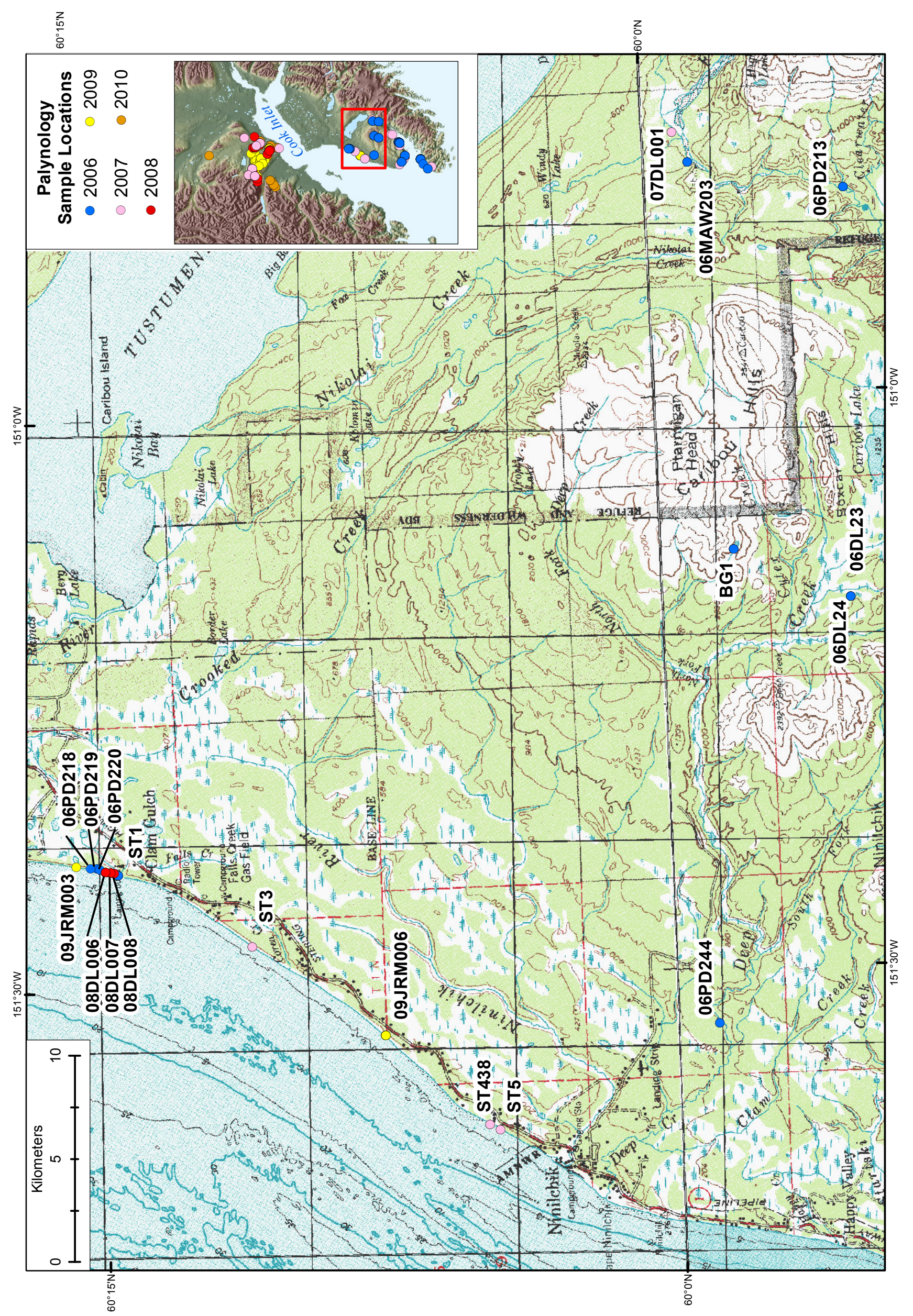

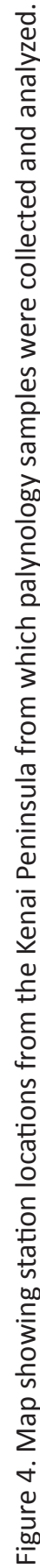




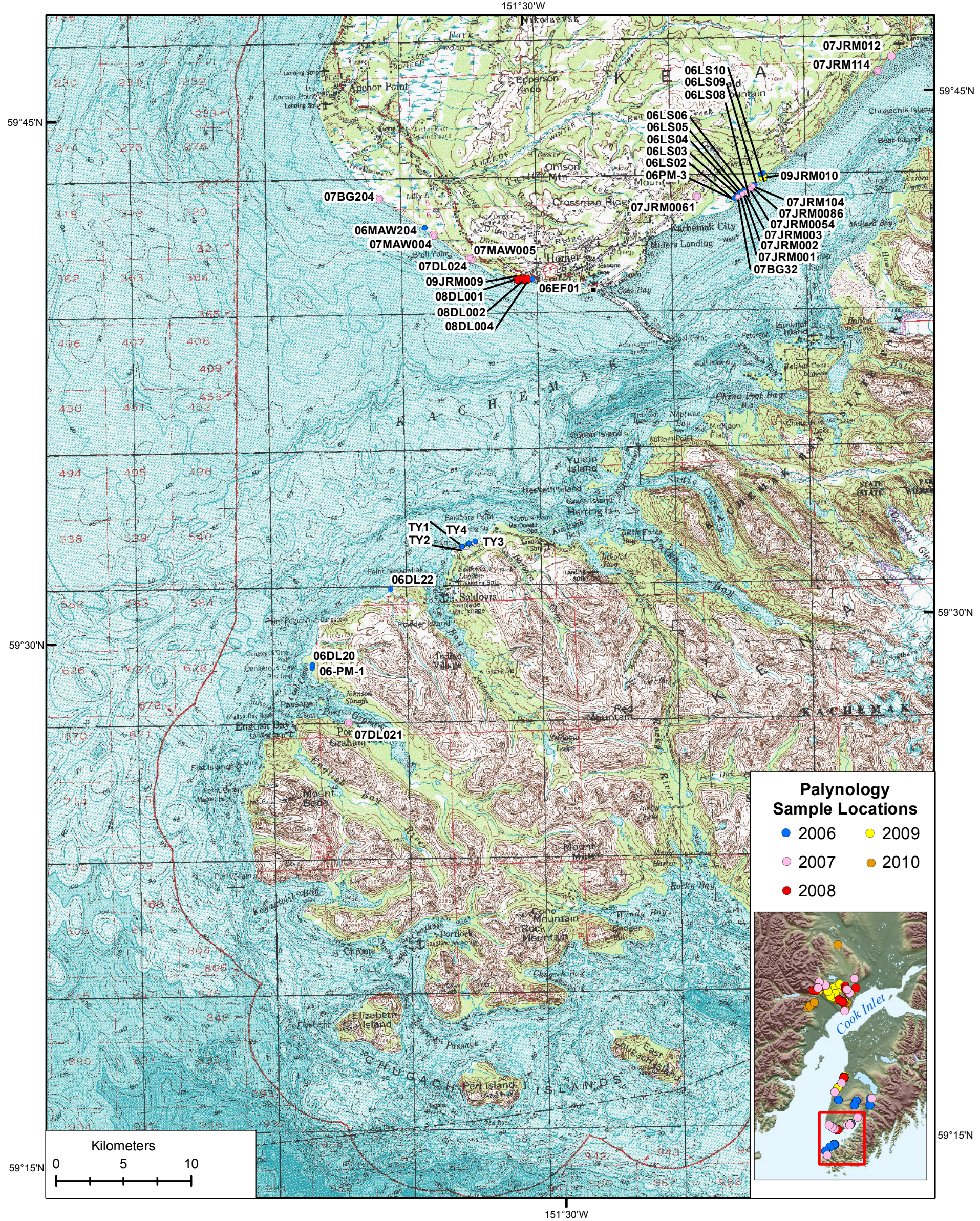

Figure 5. Map showing station locations from the southern Kenai Peninsula from which palynology samples were collected and analyzed. 
Table 1. Summary of Palynology Samples from the Cook Inlet Region.

\begin{tabular}{|c|c|c|c|c|c|}
\hline Sample & Latitude & Longitude & Geologic_Formation & Geologic_Age & Confidence_Level \\
\hline 06DL20a & 59.394550 & -151.892470 & Tyonek & Mid Miocene, Late Seldovian & not assigned \\
\hline 06DL20b & 59.394550 & -151.892470 & Tyonek & Mid Miocene, Late Seldovian & not assigned \\
\hline 06DL22c & 59.442990 & -151.786760 & Tyonek & Mid Miocene, Late Seldovian & not assigned \\
\hline 06DL23-A & 59.918330 & -151.180270 & Tyonek & Mid Miocene, Late Seldovian & not assigned \\
\hline 06DL24-0.8 & 59.918330 & -151.180270 & Tyonek & Mid Miocene, Late Seldovian & not assigned \\
\hline 06DL24-12.6 & 59.918330 & -151.180270 & Tyonek & Mid Miocene, Late Seldovian & not assigned \\
\hline 06DL24b & 59.918330 & -151.180270 & Tyonek & Mid Miocene, Late Seldovian & not assigned \\
\hline 06DL24c & 59.918330 & -151.180270 & Tyonek/Quaternary? & Mid Miocene, Late Seldovian & not assigned \\
\hline 06EF010A & 59.644200 & -151.588180 & Beluga & Late Miocene, Late Homerian & not assigned \\
\hline 06EF010B & 59.644200 & -151.588180 & Beluga & Late Miocene, Late Homerian & not assigned \\
\hline 06LS02 & 59.692490 & -151.317180 & Beluga & E. Pliocene, E. Clamgulchian & not assigned \\
\hline 06LS03 & 59.693810 & -151.313000 & Beluga & E. Pliocene, E. Clamgulchian & not assigned \\
\hline 06LS04 & 59.695070 & -151.307920 & Beluga & E. Pliocene, E. Clamgulchian & not assigned \\
\hline 06LS05 & 59.696120 & -151.303530 & Beluga & E. Pliocene, E. Clamgulchian & not assigned \\
\hline 06LS06 & 59.692000 & -151.294680 & Beluga & E. Pliocene, E. Clamgulchian & not assigned \\
\hline 06LS08 & 59.701300 & -151.290250 & Beluga & E. Pliocene, E. Clamgulchian & not assigned \\
\hline 06LS09 & 59.706360 & -151.277830 & Beluga & E. Pliocene, E. Clamgulchian & not assigned \\
\hline 06LS10 & 59.708670 & -151.277830 & Beluga & E. Pliocene, E. Clamgulchian & not assigned \\
\hline 06MAW203-34a & 59.980660 & -150.796420 & Sterling/Quaternary? & E. Pliocene, E. Clamgulchian & not assigned \\
\hline 06MAW203-54a & 59.980660 & -150.796420 & Sterling/Quaternary? & E. Pliocene, E. Clamgulchian & not assigned \\
\hline 06MAW203-60a & 59.980660 & -150.796420 & Sterling/Quaternary? & E. Pliocene, E. Clamgulchian & not assigned \\
\hline 06MAW204a & 59.680850 & -151.726330 & Beluga & Late Miocene, Late Homerian & not assigned \\
\hline 06MAW204b & 59.680850 & -151.726330 & Beluga & Late Miocene, Late Homerian & not assigned \\
\hline 06MAW204c & 59.680850 & -151.726330 & Beluga & Late Miocene, Late Homerian & not assigned \\
\hline 06MAW204d & 59.680850 & -151.726330 & Beluga & Late Miocene, Late Homerian & not assigned \\
\hline 06PD213-22.5A & 59.913620 & -150.823700 & Beluga/Sterling? & E. Pliocene, E. Clamgulchian & not assigned \\
\hline 06PD213-30.5a & 59.913620 & -150.823700 & Beluga/Sterling? & E. Pliocene, E. Clamgulchian & not assigned \\
\hline 06PD213-7a & 59.913620 & -150.823700 & Beluga/Sterling? & E. Pliocene, E. Clamgulchian & not assigned \\
\hline 06PD218a & 60.252590 & -151.391720 & Sterling & E. Pliocene, E. Clamgulchian & not assigned \\
\hline 06PD219a & 60.248650 & -151.392910 & Sterling/Quaternary? & E. Pliocene, E. Clamgulchian & not assigned \\
\hline 06PD220-23 & 60.249630 & -151.392000 & Sterling/Quaternary? & E. Pliocene, E. Clamgulchian & not assigned \\
\hline 06PD220-40.2 & 60.249630 & -151.392000 & Sterling/Quaternary? & E. Pliocene, E. Clamgulchian & not assigned \\
\hline 06PD244-1a & 59.982500 & -151.547210 & Sterling & E. Pliocene, E. Clamgulchian & not assigned \\
\hline 06PD244-25.3b & 59.982500 & -151.547210 & Sterling & E. Pliocene, E. Clamgulchian & not assigned \\
\hline 06PM-1-1 & 59.394800 & -151.892500 & Beluga & Mid Miocene, Late Seldovian & not assigned \\
\hline 06PM-1-2 & 59.394800 & -151.892500 & Beluga & Mid Miocene, Late Seldovian & not assigned \\
\hline $06 \mathrm{PM}-1-3$ & 59.394800 & -151.892500 & Beluga & Mid Miocene, Late Seldovian & not assigned \\
\hline 06PM-3-10 & 59.693380 & -151.313410 & Beluga & Late-Mid Miocene, E. Homerian & not assigned \\
\hline 06PM-3-11 & 59.693380 & -151.313410 & Beluga & Late-Mid Miocene, E. Homerian & not assigned \\
\hline 06PM-3-12 & 59.693380 & -151.313410 & Beluga & Late-Mid Miocene, E. Homerian & not assigned \\
\hline 06PM-3-17 & 59.693380 & -151.313410 & Beluga & Late-Mid Miocene, E. Homerian & not assigned \\
\hline 06PM-3-7 & 59.693380 & -151.313410 & Beluga & Late-Mid Miocene, E. Homerian & not assigned \\
\hline 06PM-3-9 & 59.693380 & -151.313410 & Beluga & Late-Mid Miocene, E. Homerian & not assigned \\
\hline 07DL001-b & 59.986890 & -150.769350 & Sterling & Pleistocene, above Clamgulchian & high \\
\hline 07DL076c & 61.01714 & -151.32707 & Beluga & Late Miocene & low-medium \\
\hline 07DL080-1.8-3.1a & 61.29171 & -151.93504 & West Foreland & Middle Eocene & medium \\
\hline 07DL080-25.8a & 61.29171 & -151.93504 & West Foreland & Middle Eocene & high \\
\hline $07 D L 080-36.0 \mathrm{~b}$ & 61.29171 & -151.93504 & West Foreland & Middle Eocene & high \\
\hline 07DL080-44.0 & 61.29171 & -151.93504 & West Foreland & Middle Eocene & high \\
\hline 07DL080-59.0a & 61.29171 & -151.93504 & West Foreland & Late Eocene & medium \\
\hline 07DL084-1.6a & 61.31741 & -151.75624 & Tyonek & late Middle Miocene & high \\
\hline 07DL084-13.5a & 61.31741 & -151.75624 & Tyonek & late Middle Miocene & high \\
\hline 07DL084-23.0a & 61.31741 & -151.75624 & Tyonek & late Middle Miocene & high \\
\hline 07DL084-29a & 61.31741 & -151.75624 & Tyonek & late Middle Miocene & high \\
\hline 07DL084-4.0 a & 61.31741 & -151.75624 & Tyonek & late Middle Miocene & high \\
\hline 07DL084-9.5a & 61.31741 & -151.75624 & Tyonek & late Middle Miocene & high \\
\hline 07DL085-06 & 61.21909 & -151.17781 & Beluga & early Late Miocene & high \\
\hline 07DL085-35 & 61.21909 & -151.17781 & Beluga & Late Miocene & medium \\
\hline 07DL085-44.0 & 61.21909 & -151.17781 & Beluga & early Late Miocene & high \\
\hline 07DL085-45.7b & 61.21734 & -151.17934 & Beluga & early Late Miocene & high \\
\hline 07DL085-49.1a & 61.21734 & -151.17934 & Beluga & early Late Miocene & high \\
\hline 07DL085-64 & 61.21909 & -151.17781 & Beluga & early Late Miocene & high \\
\hline 07DL085-90a & 61.21909 & -151.17781 & Beluga & early Late Miocene & high \\
\hline 07DL086-1.8a & 61.26407 & -151.2429 & Beluga & late Middle Miocene & high \\
\hline
\end{tabular}




\begin{tabular}{|c|c|c|c|c|c|}
\hline Sample & Latitude & Longitude & Geologic_Formation & Geologic_Age & Confidence_Level \\
\hline 07DL086-27.0 & 61.26399 & -151.24214 & Beluga & late Middle Miocene & high \\
\hline 07JRM012-70 & 59.78246 & -151.10204 & Sterling & Early Pliocene, Lower Clamgulchia & high \\
\hline 7JRM011-25.4-24.7 & 59.77309 & -151.12128 & Sterling/Beluga? & Early Pliocene, Lower Clamgulchia & high \\
\hline 7JRM010-34.9-33.9 & 59.69879 & -151.29545 & Sterling/Beluga? & Early Pliocene, Lower Clamgulchia & high \\
\hline 7JRM009-18.0-20.C & 59.70012 & -151.29219 & Sterling/Beluga? & Early Pliocene, Lower Clamgulchia & high \\
\hline 7JRM008-26.5-27.d & 59.6962 & -151.30368 & Sterling/Beluga? & Early Pliocene, Lower Clamgulchia & high \\
\hline 7JRM006-11.8-13.C & 59.69522 & -151.86672 & Sterling/Beluga? & Early Pliocene, Lower Clamgulchia & high \\
\hline 7JRM005-24.0-22.5 & 59.69517 & -151.30707 & Sterling/Beluga? & Early Pliocene, Lower Clamgulchial & high \\
\hline 07JRM003-3.5-4.5a & 59.69379 & -151.3125 & Sterling/Beluga? & Early Pliocene, Lower Clamgulchia & high \\
\hline 07JRM002-4.5-5.5a & 59.69376 & -151.31279 & Sterling/Beluga? & Early Pliocene, Lower Clamgulchia & high \\
\hline 07JRM001-1.7-3.5a & 59.69343 & -151.31347 & Sterling/Beluga? & Early Pliocene, Lower Clamgulchia & medium \\
\hline 07EF024-TK6 & 61.21909 & -151.17781 & Beluga & early Late Miocene & medium \\
\hline 08BG184b & 61.123050 & -151.353580 & Tyonek & Late Pliocene & high \\
\hline 08BG185b & 61.116980 & -151.342820 & Tyonek & Pliocene & high \\
\hline 08BG186b & 61.112790 & -151.339290 & Tyonek & Late Miocene & high \\
\hline 08BG187b & 61.224610 & -151.217180 & Tyonek & Possibly late Middle Miocene & high \\
\hline 08BG192c & 61.267430 & -152.001330 & West Foreland & $\begin{array}{l}\text { Early Oligocene, possibly Latest } \\
\text { Eocene }\end{array}$ & high \\
\hline 08BG219a & 61.304090 & -151.285930 & West Foreland & Late Eocene, Early Oligocene? & high \\
\hline 08DL050-0.0 & 61.147940 & -151.437180 & Tyonek & late Middle Miocene & high \\
\hline 08DL050-24.5 & 61.147940 & -151.437180 & Tyonek & late Middle Miocene & moderate \\
\hline 08DL052.19 & 61.276060 & -151.275050 & West Foreland & Late Eocene? & moderate \\
\hline 08DL052-7.0a & 61.276060 & -151.275050 & West Foreland & Middle to Late Eocene? & moderate \\
\hline 08DL054-48.8a & 61.273940 & -152.025620 & West Foreland & Early Oligocene to Late Eocene? & moderate \\
\hline 08DL055-0.0 & 61.262270 & -151.239880 & Tyonek & Middle Miocene & high \\
\hline 08DL055-101.0 & 61.262270 & -151.239880 & Tyonek & Middle Miocene & high \\
\hline 08DL055-57.2 & 61.262270 & -151.239880 & Tyonek & Middle Miocene & high \\
\hline 08DL055-81.0 & 61.262270 & -151.239880 & Tyonek & Middle Miocene & high \\
\hline 08DL056-10.0 & 61.264150 & -151.992483 & West Foreland & Middle Eocene & high \\
\hline 08EF015 & 61.309760 & -151.848140 & not assigned & Late Pliocene, possibly Pleistocene & very low \\
\hline 08MAW202-13.7a & 61.123270 & -151.376590 & Tyonek & Mid Miocene & moderate \\
\hline 08MAW202-5.0a & 61.123270 & -151.376590 & Tyonek & Late Pliocene & moderate \\
\hline 08MAW205-1.9a & 61.263930 & -151.063780 & not assigned & late Middle Miocene & high \\
\hline 08MAW210-2.7a & 61.276060 & -151.275010 & West Foreland & Early Pliocene & low \\
\hline 08MAW211-7.0a & 61.276400 & -151.277150 & West Foreland & early Late Oligocene & moderate \\
\hline 08MAW213-48.7a & 61.278850 & -151.025820 & West Foreland & Middle Eocene & high \\
\hline 09BG464b-a & 61.26566 & -151.26103 & not assigned & Middle to Late Eocene & high \\
\hline 09DL027-10.0a & 61.29063 & -151.29332 & West Foreland & Late Eocene? & high \\
\hline 09DL028-1.3a & 61.17379 & -151.57197 & West Foreland & Late Eocene? & high \\
\hline 09DL028-23.4a & 61.17379 & -151.57197 & West Foreland & Middle to Late Eocene & high \\
\hline 09DL030-0.6a & 61.26697 & -151.93033 & West Foreland & Middle to Late Eocene & high \\
\hline 09DL030-1.8a & 61.26697 & -151.93033 & West Foreland & Late Eocene & high \\
\hline 09DL033-0-1.5a & 61.35918 & -151.90815 & Tyonek & Late Eocene & high \\
\hline 09DL033-15.7a & 61.35918 & -151.90815 & Tyonek & Late Eocene & high \\
\hline 09JRM100-1 & 61.12356 & -151.30971 & Beluga & early Late Miocene & high \\
\hline 09JRM100-1.5 & 61.12356 & -151.30971 & Beluga & early Late Miocene & high \\
\hline 09JRM100-10 & 61.12356 & -151.30971 & Beluga & early Late Miocene & high \\
\hline 09JRM100-11.0 & 61.12353 & -151.3096 & Beluga & Late Miocene & high \\
\hline 09JRM100-12.55 & 61.12353 & -151.3096 & Beluga & Late Miocene & high \\
\hline 09JRM100-15.1 & 61.12353 & -151.3096 & Beluga & Late Miocene & high \\
\hline 09JRM100-15.2 & 61.12353 & -151.3096 & Beluga & Late Miocene & high \\
\hline 09JRM100-2 & 61.12356 & -151.30971 & Beluga & early Late Miocene & high \\
\hline 09JRM100-20.0 & 61.12353 & -151.3096 & Beluga & Late Miocene & high \\
\hline 09JRM100-23.0 & 61.12353 & -151.3096 & Beluga & Late Miocene & high \\
\hline 09JRM100-29.0 & 61.12353 & -151.3096 & Beluga & Late Miocene & high \\
\hline 09JRM100-29.5 & 61.12353 & -151.3096 & Beluga & Late Miocene & high \\
\hline 09JRM100-31.5 & 61.12353 & -151.3096 & Beluga & Late Miocene & high \\
\hline 09JRM100-32.25 & 61.12353 & -151.3096 & Beluga & Late Miocene & high \\
\hline 09JRM100-32.5 & 61.12353 & -151.3096 & Beluga & Late Miocene & high \\
\hline 09JRM100-33.0 & 61.12353 & -151.3096 & Beluga & Late Miocene & high \\
\hline 09JRM100-35.0 & 61.12353 & -151.3096 & Beluga & Late Miocene & high \\
\hline 09JRM100-38.0 & 61.12353 & -151.3096 & Beluga & Late Miocene & medium \\
\hline 09JRM100-40.7 & 61.12356 & -151.30971 & Beluga & Late Miocene & moderate \\
\hline 09JRM100-43 & 61.12356 & -151.30971 & Beluga & Late Miocene & moderate \\
\hline 09JRM100-46 & 61.12356 & -151.30971 & Beluga & Late Miocene & moderate \\
\hline 09JRM100-47 & 61.12356 & -151.30971 & Beluga & Late Miocene & moderate \\
\hline
\end{tabular}




\begin{tabular}{|c|c|c|c|c|c|}
\hline Sample & Latitude & Longitude & Geologic_Formation & Geologic_Age & Confidence_Level \\
\hline 09JRM100-5 & 61.12356 & -151.30971 & Beluga & early Late Miocene & high \\
\hline 09JRM100-5.5 & 61.12356 & -151.30971 & Beluga & early Late Miocene & high \\
\hline 09JRM100-6 & 61.12356 & -151.30971 & Beluga & early Late Miocene & high \\
\hline 09JRM100-6.5 & 61.12356 & -151.30971 & Beluga & early Late Miocene & high \\
\hline 09JRM100-7 & 61.12356 & -151.30971 & Beluga & early Late Miocene & high \\
\hline 09MAW302a & 61.2877 & -151.53159 & Hemlock/Tyonek? & Early Miocene - Late Oligocene & medium \\
\hline 09MAW303a & 61.29188 & -151.52861 & Hemlock/Tyonek? & Early Miocene - Late Oligocene & medium \\
\hline 09MAW306 & 61.29413 & -151.52089 & Hemlock/Tyonek? & Early Miocene - Late Oligocene & low \\
\hline 09MAW308a & 61.28328 & -151.60304 & Hemlock/Tyonek? & Early Miocene & moderate \\
\hline 09MAW312a & 61.28196 & -151.59036 & Hemlock/Tyonek? & $\begin{array}{c}\text { Early Miocene - Late Oligocene, } \\
\text { possibly Middle Eocene }\end{array}$ & low-medium \\
\hline 09MAW319a & 61.27144 & -151.46733 & West Foreland & Middle Eocene & high \\
\hline 09MAW321a & 61.28325 & -151.42183 & West Foreland & Middle Eocene & low \\
\hline 09MAW324a & 61.32767 & -151.4209 & Hemlock & Late Oligocene & low-medium \\
\hline 09MAW330a & 61.26768 & -151.56367 & Hemlock/Tyonek? & Late Oligocene & low-medium \\
\hline 09MAW345a & 61.20785 & -151.562 & Tyonek & late Middle Miocene & high \\
\hline 09MAW346a & 61.20784 & -151.56561 & Tyonek & late Middle Miocene & high \\
\hline 09MAW347a & 61.20948 & -151.56361 & Tyonek & late Middle Miocene & high \\
\hline 09MAW359a & 61.22662 & -151.59159 & West Foreland & Middle Eocene & moderate-high \\
\hline 09MAW360a & 61.22841 & -151.59436 & West Foreland & Oligocene to Middle Eocene & moderate \\
\hline 09PD245b & 61.28949 & -151.79686 & Tyonek & $\begin{array}{c}\text { early Late Miocene-late Middle } \\
\text { Miocene }\end{array}$ & moderate \\
\hline 09PD248b & 61.27774 & -151.71515 & Tyonek & late Middle Miocene & high \\
\hline 09PD278a & 61.22113 & -151.53584 & Hemlock/Tyonek? & Early (mid) Oligocene & moderate \\
\hline 09PD279a & 61.22257 & -151.53107 & Hemlock/Tyonek? & Early (mid) Oligocene & moderate \\
\hline 09PD303a & 61.28702 & -151.31953 & West Foreland & Middle Eocene & high \\
\hline 09PD308a & 61.26453 & -151.26039 & Tyonek & Middle Miocene & high \\
\hline 09TMH-335B & 61.30284 & -151.82562 & Hemlock/Tyonek? & early Middle Miocene & high \\
\hline 09TMH364b & 61.20236 & -151.66896 & Hemlock/Tyonek? & Early Miocene & moderate \\
\hline 09TMH367a & 61.19684 & -151.65691 & Hemlock/Tyonek? & Early Miocene & moderate \\
\hline 09TMH411 & 61.24491 & -151.70836 & Hemlock/Tyonek? & Middle Miocene & high \\
\hline 10BG-402A & 61.31646 & -151.76685 & Tyonek & Middle Miocene & moderate-high \\
\hline 10BG-404A & 61.26308 & -151.24252 & Tyonek & Middle Miocene & moderate-high \\
\hline 10BG-406A & 61.15346 & -151.51147 & Tyonek & Middle Miocene & moderate-high \\
\hline 10BG-409A & 61.26608 & -151.26408 & Hemlock? & Early Miocene & moderate \\
\hline 10BG-416A & 61.27428 & -151.26744 & Tyonek & Middle Miocene & moderate-low \\
\hline 10BG-417A & 61.27522 & -151.27048 & Hemlock? & Early Miocene-latest Oligocene? & moderate \\
\hline 10BG-425A & 61.07438 & -152.19998 & Tyonek? & Middle Miocene & moderate-low \\
\hline 10BG-448A & 61.10042 & -152.1097 & Tyonek? & Early Miocene & moderate \\
\hline 10BG-463A & 61.22773 & -151.22552 & Tyonek & Middle Miocene & moderate-high \\
\hline 10BG-478A & 61.78238 & -151.42191 & Sterling? & Middle Miocene & moderate \\
\hline 10DJM-102A & 61.14694 & -151.44104 & Tyonek & early Late Miocene & moderate \\
\hline 10DJM-113A & 61.22049 & -151.1456 & Sterling? & latest Miocene-Early Pliocene & moderate \\
\hline 10DJM-114A & 61.03226 & -151.30663 & Beluga & early Late Miocene & moderate \\
\hline 10DJM-116B & 61.03394 & -151.30157 & Beluga & latest Miocene & moderate \\
\hline 10DJM-62A & 61.14941 & -151.48997 & Tyonek & Middle Miocene & moderate \\
\hline 10DJM-72A & 61.24105 & -151.23723 & Tyonek & Middle Miocene & moderate \\
\hline 10DJM-74A & 61.23261 & -151.21729 & Beluga? & Middle Miocene & moderate \\
\hline 10DJM-96A & 61.11749 & -151.28677 & Beluga & early Late Miocene & moderate \\
\hline 10DL-040A & 61.30844 & -151.8237 & Hemlock? & early Middle Miocene & moderate \\
\hline 10DL-040B & 61.30844 & -151.8237 & Hemlock? & early Middle Miocene & high \\
\hline 10DL-040C & 61.30844 & -151.8237 & Hemlock? & early Middle Miocene & high \\
\hline 10DL-043E & 61.2095 & -151.56357 & Hemlock? & early Middle Miocene & low-moderate \\
\hline 10TMH-227A & 61.22345 & -151.2057 & Beluga? & latest Miocene & moderate-high \\
\hline 10TMH-230A & 61.23698 & -151.23288 & Beluga? & latest Miocene & moderate-high \\
\hline 10TMH-234A & 61.23576 & -151.23547 & Beluga? & Middle Miocene & moderate-high \\
\hline 10TMH-238B & 61.23412 & -151.24075 & Beluga? & latest Miocene & moderate-high \\
\hline 10TMH-245B & 61.30422 & -151.28571 & Tyonek? & Middle Miocene & moderate-high \\
\hline 10TMH-248A & 61.13046 & -151.39043 & Tyonek & Late Miocene & low-moderate \\
\hline 10TMH-250A & 61.11292 & -151.33788 & Beluga & early Late Miocene & moderate \\
\hline 10TMH-251A & 61.11618 & -151.32272 & Beluga & Middle Miocene & moderate-high \\
\hline 10TMH-253A & 61.11871 & -151.30269 & Beluga & Middle Miocene & moderate-high \\
\hline 10TMH-256A & 61.10861 & -151.24725 & Beluga & Middle Miocene & moderate-high \\
\hline 10TMH-257E & 61.11695 & -151.34256 & Tyonek? & Middle Miocene & moderate-low \\
\hline 10TMH-265A & 61.10003 & -152.13268 & Tyonek & Middle Miocene & moderate-high \\
\hline 10TMH-270A & 61.30535 & -151.82275 & Tyonek & Middle Miocene & moderate-high \\
\hline
\end{tabular}




\begin{tabular}{|c|c|c|c|c|c|}
\hline Sample & Latitude & Longitude & Geologic_Formation & Geologic_Age & Confidence_Level \\
\hline 10TMH-271B & 61.30762 & -151.8226 & Tyonek? & early Middle Miocene & high \\
\hline 10TMH-272A & 61.30914 & -151.82076 & Tyonek? & early Middle Miocene & high \\
\hline 10TMH-273A & 61.09037 & -152.14404 & Tyonek & Middle Miocene & moderate-high \\
\hline 10TMH-274A & 61.0884 & -152.14334 & Tyonek & early Late Miocene & low \\
\hline 10TMH-276B & 61.12384 & -152.03677 & Tyonek & Middle Miocene & moderate-high \\
\hline 10TMH-282A & 61.26455 & -151.26038 & Tyonek & Middle Miocene & moderate-high \\
\hline BG1-26.3 & 59.968180 & -151.135070 & Beluga & Late Miocene, Late Homerian & not assigned \\
\hline BG1-35.7 & 59.968180 & -151.135070 & Beluga & Late Miocene, Late Homerian & not assigned \\
\hline BG1-5.1 & 59.968180 & -151.135070 & Beluga & Late Miocene, Late Homerian & not assigned \\
\hline BG1-8.1 & 59.968180 & -151.135070 & Beluga & Late Miocene, Late Homerian & not assigned \\
\hline ST1-15.4 & 60.240640 & -151.398480 & Sterling & E. Pliocene, E. Clamgulchian & not assigned \\
\hline ST1-27.7 & 60.240640 & -151.398480 & Sterling & E. Pliocene, E. Clamgulchian & not assigned \\
\hline ST1-74.5 & 60.240640 & -151.398480 & Sterling & E. Pliocene, E. Clamgulchian & not assigned \\
\hline ST1-79.9 & 60.240640 & -151.398480 & Sterling & E. Pliocene, E. Clamgulchian & not assigned \\
\hline TY1-15.8 & 59.469360 & -151.690980 & Tyonek & Mid Miocene, Late Seldovian & not assigned \\
\hline TY1-21 & 59.469360 & -151.690980 & Tyonek & Mid Miocene, Late Seldovian & not assigned \\
\hline TY1-26 & 59.469360 & -151.690980 & Tyonek & Mid Miocene, Late Seldovian & not assigned \\
\hline TY1-32 & 59.469360 & -151.690980 & Tyonek & Mid Miocene, Late Seldovian & not assigned \\
\hline TY1-4.5 & 59.469360 & -151.690980 & Tyonek & Mid Miocene, Late Seldovian & not assigned \\
\hline TY1-9.5 & 59.469360 & -151.690980 & Tyonek & Mid Miocene, Late Seldovian & not assigned \\
\hline TY2-10.8 & 59.469360 & -151.690980 & Tyonek & Mid Miocene, Late Seldovian & not assigned \\
\hline TY2-12.5 & 59.469360 & -151.690980 & Tyonek & Mid Miocene, Late Seldovian & not assigned \\
\hline TY2-17 & 59.469360 & -151.690980 & Tyonek & Mid Miocene, Late Seldovian & not assigned \\
\hline TY2-3.0 & 59.469360 & -151.690980 & Tyonek & Mid Miocene, Late Seldovian & not assigned \\
\hline TY2-4.8 & 59.469360 & -151.690980 & Tyonek & Mid Miocene, Late Seldovian & not assigned \\
\hline TY3-12.2 & 59.472720 & -151.674270 & Tyonek & Mid Miocene, Late Seldovian & not assigned \\
\hline TY3-2.0 & 59.472720 & -151.674270 & Tyonek & Mid Miocene, Late Seldovian & not assigned \\
\hline TY33-5.5 & 59.472720 & -151.674270 & Tyonek & Mid Miocene, Late Seldovian & not assigned \\
\hline TY4-10.3 & 59.471780 & -151.682620 & Tyonek & Mid Miocene, Late Seldovian & not assigned \\
\hline TY4-2.1 & 59.471780 & -151.682620 & Tyonek & Mid Miocene, Late Seldovian & not assigned \\
\hline TY4-2.9 & 59.471780 & -151.682620 & Tyonek & Mid Miocene, Late Seldovian & not assigned \\
\hline TY4-6 & 59.471780 & -151.682620 & Tyonek & Mid Miocene, Late Seldovian & not assigned \\
\hline ST3-87 & 60.183730 & -151.465910 & Sterling & $\begin{array}{l}\text { Pleistocene/Late Pliocene, } \\
\text { U. Clamgulchian or above }\end{array}$ & low \\
\hline ST4-38 & 60.083530 & -151.628860 & Sterling & Pliocene, Clamgulchian & medium \\
\hline ST5-7 & 60.079140 & -151.633730 & Sterling & Pliocene, Clamgulchian & medium \\
\hline
\end{tabular}

